\title{
Differences in Urodynamic Variables for Vesicoureteral Reflux Depending on the Neurogenic Bladder Type
}

\author{
Je Sang Lee, $\mathrm{MD}^{1,2,3}$, Bon Il Koo, $\mathrm{MD}^{1,2,4}$, Myung Jun Shin, $\mathrm{MD}^{1,2,3}$, Jae Hyeok Chang, $\mathrm{MD}^{1,2,3}$, \\ Soo-Yeon Kim, $\mathrm{MD}^{1,2,4}$, Hyun-Yoon Ko, $\mathrm{MD}^{1,2,4}$
}

\begin{abstract}
${ }^{1}$ Division of Spinal Cord Medicine, ${ }^{2}$ Department of Rehabilitation Medicine, Pusan National University School of Medicine, Yangsan; ${ }^{3}$ Medical Research Institute, Pusan National University, Busan; ${ }^{4}$ Research Institute for Convergence of Biomedical Science and Technology, Pusan National University Yangsan Hospital, Yangsan, Korea
\end{abstract}

Objective To compare the urodynamic study variables at the onset of vesicoureteral reflux (VUR) between the overactive and underactive bladders in patients with spinal cord injury who presented with VUR.

Methods A total of 28 (13 cases of detrusor overactivity and 15 detrusor underactivity) men were enrolled. We compared the urodynamic variables between the two groups; detrusor pressure and bladder compliance, the infused volume at the onset of VUR measured on a voiding cystourethrography and cystometric capacity, maximum detrusor pressure, and bladder compliance during filling cystometry were recorded.

Results At the onset of VUR, the bladder volume and compliance, except for the detrusor pressure, showed a significant difference between the two groups. The detrusor pressure, bladder volume, and bladder compliance relative to the cystometric capacity showed a significant difference between the two groups. The detrusor pressure, bladder volume, and bladder compliance at the onset of VUR relative to the cystometric bladder capacity did not show any significant difference between the two groups.

Conclusion There were differences in some variables at the onset of VUR depending on the type of neurogenic bladder. The VUR occurred at a lower capacity in neurogenic bladder with detrusor overactivity than in neurogenic bladder with detrusor underactivity at the same pressure. VUR occurred at a lower intravesical pressure compared to that known as the critical detrusor pressure $\left(\geq 40 \mathrm{~cm} \mathrm{H}_{2} \mathrm{O}\right)$ required for the development of VUR. The results of our study demonstrate that the detrusor pressure should be maintained lower than the well known effective critical detrusor pressure for the prevention and treatment of VUR.

Keywords Neurogenic urinary bladder, Vesicoureteral reflux, Spinal cord injuries, Urodynamics 


\section{INTRODUCTION}

Unless appropriately treated, neurogenic bladder after spinal cord injury would lead to bladder deformity or other complications, such as vesicoureteral reflux (VUR), hydronephrosis, and renal failure [1]. VUR has been reported to occur due to higher intravesical pressure and urethral pressure, low bladder compliance, and thickening of the bladder wall resulting in changes at the vesicoureteric junction and intramural obliquity. Untreated VUR may lead to hydronephrosis and upper urinary tract infection, and ultimately cause renal failure [2].

According to the anatomic level of the lesion, neurogenic bladder after spinal cord injury is classified as suprasacral and infrasacral neurogenic bladder. Functionally, based on the results of urodynamic study during the filling phase, neurogenic bladders can be classified into detrusor overactive or underactive [3,4]. VUR occurs regardless of the type of neurogenic bladder. However, there are no well-designed studies assessing the differences in urodynamic study variables depending on the type of neurogenic bladder.

In the present study, we compared the urodynamic study variables at the onset of VUR between overactive and underactive bladders in patients with spinal cord injury who presented with VUR. In addition, based on the results of urodynamic study at the onset of VUR, we attempted to present the baseline data and the relevant guidelines for the optimal time of voiding by intermittent catheterization for the prevention of VUR in patients with neurogenic bladder.

\section{MATERIALS AND METHODS}

Three hundred twelve patients with spinal cord injury underwent urodynamic study and voiding cystourethrography on the same day from February 2008 to June 2011. Sixty-eight patients were confirmed to have VUR based on the international grading system [5] on voiding cystourethrography. Patients with peripheral neuropathy, those with an evidence of urinary tract infection, those who had concurrent brain injury, and those who were taking drugs that may affect the intravesical pressure, such as anticholinergics or cholinergics, were excluded. Finally, a total of 28 men were enrolled in the present study. In all of the study subjects, more than three months had elapsed since the onset of spinal cord injury. Mean age of the study subjects was $51.46 \pm 10.7$ years. In these patients, a mean period of $92 \pm 103$ months had elapsed since the spinal cord injury (Table 1).

The bladder was filled with a contrast medium using the drip infusion method and the infusion was controlled by an infusion pump at a rate of $50 \mathrm{~mL} / \mathrm{min}$. This was followed by assessment of the amount of contrast medium infused until the onset of VUR. A urodynamic study was also performed on the same day as voiding cystourethrography, and it was performed using Solar Gold (MMS, Enschede, The Netherlands). For performing these procedures, saline was infused into the bladder at room temperature at a rate of $24 \mathrm{~mL} / \mathrm{min}$ using a two-lumen catheter.

Detrusor overactivity was defined according to the standardization of the 2002 International Continence Society, and it is defined as a condition in which there are spontaneous or provoked involuntary detrusor contractions [6]. There were 13 cases of detrusor overactivity and 15 cases of detrusor underactivity in this study.

The amount of contrast medium infused into the bladder at the onset of VUR was measured on the voiding cystourethrogram. The urodynamic variables, detrusor pressure and bladder compliance, the infused volume at the onset of VUR measured on a voiding cystourethrogram

Table 1. Demographic and clinical variables

\begin{tabular}{lcc}
\hline \multicolumn{1}{c}{ Variable } & $\begin{array}{c}\text { Detrusor } \\
\text { overactivity } \\
(\mathbf{n}=\mathbf{1 3})\end{array}$ & $\begin{array}{c}\text { Detrusor } \\
\text { underactivity } \\
(\mathbf{n}=\mathbf{1 5})\end{array}$ \\
\hline $\begin{array}{l}\text { Age (yr) } \\
\text { Duration of injury (mo) }\end{array}$ & $\begin{array}{c}53.15 \pm 11.0 \\
50.00 \pm 10.5\end{array}$ \\
$\begin{array}{l}\text { Neurological level of injury } \\
\text { Cervical }\end{array}$ & $6(46)$ & $7(46)$ \\
\hline Thoracic & $3(23)$ & $4(27)$ \\
\hline Lumbar and/or sacral & $4(31)$ & $4(27)$ \\
AIS & & \\
\hline A & $5(38)$ & $5(33)$ \\
\hline B & $1(8)$ & $1(7)$ \\
C & $4(31)$ & $7(47)$ \\
D & $3(23)$ & $2(13)$ \\
\hline
\end{tabular}

Values are presented as mean \pm standard deviation or number (\%).

AIS, American Spinal Injury Association impairment scale. 
and cystometric capacity, maximum detrusor pressure, and bladder compliance during filling cystometry were recorded. We compared the urodynamic variables between the bladders with detrusor overactivity and detrusor underactivity. Statistical analysis was performed with the Mann-Whitney test using SPSS ver. 18.0 (SPSS Inc., Chicago, IL, USA).

\section{RESULTS}

In the detrusor overactivity group, the detrusor pressure, bladder volume, and bladder compliance at the onset of VUR were $19.23 \pm 20.96 \mathrm{~cm} \mathrm{H}_{2} \mathrm{O}, 120.38 \pm 88.56$ $\mathrm{mL}$, and $39.12 \pm 69.79 \mathrm{~mL} / \mathrm{cm} \mathrm{H}_{2} \mathrm{O}$, respectively. In the detrusor underactivity group, the detrusor pressure, bladder volume, and bladder compliance at the onset of VUR were $10.06 \pm 9.94 \mathrm{~cm} \mathrm{H}_{2} \mathrm{O}, 282.33 \pm 172.82 \mathrm{~mL}$, and $68.60 \pm 134.57 \mathrm{~mL} / \mathrm{cm} \mathrm{H}_{2} \mathrm{O}$, respectively (Table 2).

The detrusor pressure, bladder volume, and bladder compliance during filling cystometry in the de- trusor overactivity group were $62.00 \pm 29.96 \mathrm{~cm} \mathrm{H}_{2} \mathrm{O}$, $230.61 \pm 79.42 \mathrm{~mL}$, and $12.64 \pm 21.39 \mathrm{~mL} / \mathrm{cm} \mathrm{H}_{2} \mathrm{O}$, respectively. In the detrusor underactivity group, the detrusor pressure, bladder volume, and bladder compliance during filling cystometry were $25.04 \pm 20.73 \mathrm{~cm} \mathrm{H}_{2} \mathrm{O}$, $457.06 \pm 141.93 \mathrm{~mL}$, and $33.74 \pm 39.27 \mathrm{~mL} / \mathrm{cm} \mathrm{H}_{2} \mathrm{O}$, respectively (Table 3 ). At the onset of VUR, the bladder volume and bladder compliance, except for the detrusor pressure, showed a significant difference between the two groups. The detrusor pressure, bladder volume, and bladder compliance relative to the cystometric bladder capacity showed a significant difference between the two groups $(\mathrm{p}<0.05)$. The detrusor pressure, bladder volume, and bladder compliance at the onset of VUR relative to the cystometric bladder capacity did not any significant difference between the two groups (Table 4).

\section{DISCUSSION}

Ku et al. [7] reported that the incidence of VUR after

Table 2. Comparison of urodynamic variables at the onset of vesicoureteral reflux between the two groups

\begin{tabular}{lccc}
\hline \multicolumn{1}{c}{ Variable } & Detrusor overactivity & Detrusor underactivity & p-value \\
\hline Bladder capacity $(\mathrm{mL})$ & $120.38 \pm 88.56$ & $282.33 \pm 172.82$ & 0.01 \\
Detrusor pressure $\left(\mathrm{cm} \mathrm{H}_{2} \mathrm{O}\right)$ & $19.23 \pm 20.96$ & $10.06 \pm 9.94$ & 0.61 \\
Compliance $\left(\mathrm{mL} / \mathrm{cm} \mathrm{H}_{2} \mathrm{O}\right)$ & $39.12 \pm 69.79$ & $68.60 \pm 134.57$ & 0.02 \\
\hline
\end{tabular}

Values are presented as mean \pm standard deviation.

Table 3. Comparison of urodynamic variables during filling cystometry between the two groups

\begin{tabular}{lccc}
\multicolumn{1}{c}{ Variable } & Detrusor overactivity & Detrusor underactivity & p-value \\
\hline Bladder capacity $(\mathrm{mL})$ & $230.61 \pm 79.42$ & $457.06 \pm 141.93$ & $<0.001$ \\
Detrusor pressure $\left(\mathrm{cm} \mathrm{H}_{2} \mathrm{O}\right)$ & $62.00 \pm 29.96$ & $25.04 \pm 20.73$ & 0.001 \\
Compliance $\left(\mathrm{mL} / \mathrm{cm} \mathrm{H}_{2} \mathrm{O}\right)$ & $12.64 \pm 21.39$ & $33.74 \pm 39.27$ & 0.001 \\
\hline
\end{tabular}

Values are presented as mean \pm standard deviation.

Table 4. Comparison of urodynamic ratios: the onset of vesicoureteral reflux vs. the cystometric capacity between the two groups

\begin{tabular}{lccc}
\hline & Detrusor overactivity & Detrusor underactivity & p-value \\
\hline Bladder capacity ratio (a:A) & $0.67 \pm 0.54$ & $0.63 \pm 0.35$ & 0.52 \\
Detrusor pressure ratio (b:B) & $0.38 \pm 0.49$ & $0.42 \pm 0.28$ & 0.27 \\
Compliance ratio (c:C) & $3.12 \pm 3.89$ & $1.58 \pm 1.00$ & 0.33 \\
\hline
\end{tabular}

a, bladder capacity $(\mathrm{mL})$; b, detrusor pressure $\left(\mathrm{cm} \mathrm{H}_{2} \mathrm{O}\right)$; c, compliance $\left(\mathrm{mL} / \mathrm{cm} \mathrm{H}_{2} \mathrm{O}\right)$ at the onset of vesicoureteral reflux.

A, bladder capacity $(\mathrm{mL})$; B, detrusor pressure $\left(\mathrm{cm} \mathrm{H}_{2} \mathrm{O}\right)$; C, compliance $\left(\mathrm{mL} / \mathrm{cm} \mathrm{H}_{2} \mathrm{O}\right)$ during filling cystometry. 
spinal cord injury was $15.1 \%$. Kaufman et al. [8] reported that the prevalence of VUR was $56 \%$ in patients with encephalocele whose intravesical pressure was relatively higher. Gerridzen et al. [9] reported that there were complications in the upper urinary tract among patients whose intravesical pressure was higher than $115 \mathrm{~cm} \mathrm{H}_{2} \mathrm{O}$. McGuire et al. [10] demonstrated that there was a significant correlation between the leak point pressure of $>40 \mathrm{~cm} \mathrm{H}_{2} \mathrm{O}$ and the risk of complications in the upper urinary tract. In addition, Hackler et al. [11] reported that among the 254 studied patients with spinal cord injury, the incidence of VUR was relatively higher in the low compliance group. Many studies have been conducted to examine the incidence of VUR following spinal cord injury, the causative factors of VUR, and the urodynamic study variables. However, there has been no study assessing the urodynamic variables at the onset of VUR in patients with spinal cord injury who had VUR.

In the current study, we compared the urodynamic study variables at the onset of VUR depending on the type of neurogenic bladder. Our results showed that the VUR occurred at a relatively lower capacity in the neurogenic bladder with detrusor overactivity than in the neurogenic bladder with detrusor underactivity. The bladder compliance at the onset of VUR was higher in the neurogenic bladder with detrusor underactivity. The detrusor pressure did not show any significant difference between the two types of neurogenic bladder. The detrusor pressure was $19.23 \pm 20.96 \mathrm{~cm} \mathrm{H}_{2} \mathrm{O}$ in the detrusor overactivity group and $10.06 \pm 9.94 \mathrm{~cm} \mathrm{H}_{2} \mathrm{O}$ in the detrusor underactivity group. This indicates that the VUR occurred at a lower pressure than that indicated by the well-known urodynamic study variables: leak point pressure of $40 \mathrm{~cm} \mathrm{H}_{2} \mathrm{O}$ [10], maximal detrusor pressure of $70 \mathrm{~cm} \mathrm{H}_{2} \mathrm{O}$ [12].

Both urodynamic study and voiding cystourethrography are routinely performed in different conditions from the physiological natural bladder filling. It can therefore be inferred that the results of these studies would be different from those obtained in physiological conditions. Filling rate of the filling media would affect the bladder compliance and the maximal cystometric capacity. Even at a lower filling rate of $<20 \mathrm{~mL} / \mathrm{min}$, the detrusor pressure during bladder filling would be increased compared with that during natural bladder filling [13]. It is also known that the bladder compliance would be decreased with an increase in the filling rate [14]. In the current study, at a filling rate of $24 \mathrm{~mL} / \mathrm{min}$, we attempted to minimize the effects that may be generated during artificial filling. In addition, when a urodynamic study was performed twice consecutively, the bladder volume and compliance were increased during the second session as compared with those in the first session of bladder filling with saline [15]. In the current study, instead of a videoassisted urodynamic study, we presented the results of voiding cystourethrography and urodynamic study. This is one of the limitations of the present study that there is a lack of synchrony. Further studies are warranted to minimize these effects by performing a video-assisted urodynamic study.

In the current study, we performed urinalysis to exclude urinary tract infection that can affect bladder compliance and detrusor pressure on the same day as urodynamic study and voiding cystourethrography $[16,17]$.

We conducted the current study only in male patients with spinal cord injury. It is known that there are sex-related differences in the VUR, and the incidence of VUR is relatively higher in women than in men [18]. This might be due to the anatomical differences, higher incidence of urinary tract infection, and a higher mean maximum detrusor pressure in women.

With regard to the type of neurogenic bladder depending on the level of spinal cord injury, Bors and Comarr [19] classified neurogenic bladder into the upper motor neuron bladders and lower motor neuron bladders depending on whether the level of injury was suprasacral or infrasacral. These authors reported that there was a higher prevalence of detrusor overactivity in cases of upper motor neuron injury and detrusor underactivity in cases of lower motor neuron injury. As compared with the other sites of spinal cord injury, the incidence of VUR has been reported to be relatively higher in cases of injury to the neurological level of T10-L2 [20]. However, there was no significant difference in the type of neurogenic bladder depending on the sites of spinal cord injury in our study.

It is known that the development of VUR is mainly due to factors, such as high leak point pressure [10] and high maximum detrusor pressure [12]. There are no established criteria for the optimal detrusor pressure for preventing injury to the upper urinary tract in patients with VUR.

We found that there was a significant difference in the results of the urodynamic study at the onset of VUR de- 
pending on the type of neurogenic bladder. It was also observed that the VUR occurred at a lower capacity in the neurogenic bladder with detrusor overactivity than in the neurogenic bladder with detrusor underactivity. Our results also showed that VUR occurred at a detrusor pressure of $<40 \mathrm{~cm} \mathrm{H}_{2} \mathrm{O}$, the leak point pressure which has been recognized so far as the critical level for the development of VUR in both types of neurogenic bladder.

In conclusion, there were differences in some urodynamic variables at the onset of VUR depending on the type of neurogenic bladder. The VUR occurred at a lower capacity in the neurogenic bladder with detrusor overactivity than in the neurogenic bladder with detrusor underactivity at the same pressure. VUR occurred at a lower intravesical pressure compared to that known as the critical detrusor pressure required for the development of VUR. Further studies are warranted to examine the role of detrusor pressure in order to effectively prevent the development of VUR. The results of our present study demonstrate that the detrusor pressure should be maintained lower than the well-known effective critical detrusor pressure for the prevention and treatment of VUR.

\section{CONFLICT OF INTEREST}

No potential conflict of interest relevant to this article was reported.

\section{ACKNOWLEGMENTS}

This study was partly supported by a 2013 research grant from Pusan National University Yangsan Hospital.

\section{REFERENCES}

1. Talbot HS, Bunts RC. Late renal changes in paraplegia; hydronephrosis due to vesico-ureteral reflux. J Urol 1949;61:870-82.

2. Arant BS Jr. Vesicoureteric reflux and renal injury. Am J Kidney Dis 1991;17:491-511.

3. Madersbacher $\mathrm{H}$. The various types of neurogenic bladder dysfunction: an update of current therapeutic concepts. Paraplegia 1990;28:217-29.

4. Madersbacher H, Wyndaele JJ, Igawa Y, Chancellor M, Chartier-Kastler E, Kovindha A. Conservative management in neuropathic urinary incontinence.
In: Abrams P, Cardozo L, Khoury S, Wein A, editors. Incontinence: 2nd international consultation on incontinence. Plymouth: Health Publication Ltd.; 2002. p. 697-754.

5. Lebowitz RL, Olbing H, Parkkulainen KV, Smellie JM, Tamminen-Mobius TE. International system of radiographic grading of vesicoureteric reflux: International Reflux Study in Children. Pediatr Radiol 1985;15:1059.

6. Abrams P, Cardozo L, Fall M, Griffiths D, Rosier P, Ulmsten $U$, et al. The standardisation of terminology of lower urinary tract function: report from the Standardisation Sub-committee of the International Continence Society. Am J Obstet Gynecol 2002;187:116-26.

7. Ku JH, Choi WJ, Lee KY, Jung TY, Lee JK, Park WH, et al. Complications of the upper urinary tract in patients with spinal cord injury: a long-term follow-up study. Urol Res 2005;33:435-9.

8. Kaufman AM, Ritchey ML, Roberts AC, Rudy DC, McGuire EJ. Decreased bladder compliance in patients with myelomeningocele treated with radiological observation. J Urol 1996;156:2031-3.

9. Gerridzen RG, Thijssen AM, Dehoux E. Risk factors for upper tract deterioration in chronic spinal cord injury patients. J Urol 1992;147:416-8.

10. McGuire EJ, Woodside JR, Borden TA, Weiss RM. Prognostic value of urodynamic testing in myelodysplastic patients. J Urol 1981;126:205-9.

11. Hackler RH, Hall MK, Zampieri TA. Bladder hypocompliance in the spinal cord injury population. J Urol 1989;141:1390-3.

12. Wyndaele JJ. Urology in spinal cord injured patients. Paraplegia 1987;25:267-9.

13. Dmochowski R. Cystometry. Urol Clin North Am 1996;23:243-52.

14. Styles RA, Neal DE, Griffiths CJ, Ramsden PD. Longterm monitoring of bladder pressure in chronic retention of urine: the relationship between detrusor activity and upper tract dilatation. J Urol 1988;140:330-4.

15. Downey JA, Myers SJ, Gonzalez EG, Lieberman JS. The physiological basis of rehabilitation medicine. 2nd ed. Boston: Butterworth-Heinemann; 1994.

16. Caione P, Villa M, Capozza N, De Gennaro M, Rizzoni G. Predictive risk factors for chronic renal failure in primary high-grade vesico-ureteric reflux. BJU Int 2004;93:1309-12. 
17. Soylu A, Demir BK, Turkmen M, Bekem O, Saygi M, Cakmakci $\mathrm{H}$, et al. Predictors of renal scar in children with urinary infection and vesicoureteral reflux. Pediatr Nephrol 2008;23:2227-32.

18. Lipsky H, Chisholm GD. Primary vesico-ureteric reflux in adults. Br J Urol 1971;43:277-83.
19. Bors E, Comarr AE. Vesico-ureteral reflux in paraplegic patients. J Urol 1952;68:691-8.

20. Suzuki T, Ushiyama T. Vesicoureteral reflux in the early stage of spinal cord injury: a retrospective study. Spinal Cord 2001;39:23-5. 